\title{
A Set of Data Mining Models to Classify Credit Cardholder Behavior
}

\author{
Gang Kou ${ }^{1}$, Yi Peng ${ }^{1}$, Yong $\mathrm{Shi}^{1}$, and Weixuan $\mathrm{Xu}^{2}$ \\ ${ }^{1}$ College of Information Science and Technology \\ University of Nebraska at Omaha \\ Omaha, NE 68182, USA \\ yshi@unomaha.edu \\ ${ }^{2}$ Institute of Policy and Management \\ Chinese Academy of Sciences \\ Beijing, 100080, China \\ wxu@mail.casipm.ac.cn
}

\begin{abstract}
In this paper, we present a set of classification models by using multiple criteria linear programming (MCLP) to discover the various behaviors of credit cardholders. In credit card portfolio management, predicting the cardholder's spending behavior is a key to reduce the risk of bankruptcy. Given a set of predicting variables (attributes) that describes all possible aspects of credit cardholders, we first present a set of general classification models that can theoretically handle any size of multiple-group cardholders' behavior problems. Then, we implement the algorithm of the classification models by using SAS and Linux platforms. Finally, we test the models on a special case where the cardholders' behaviors are predefined as five classes: (i) bankrupt charge-off; (ii) non-bankrupt charge-off; (iii) delinquent; (iv) current and (v) outstanding on a real-life credit card data warehouse. As a part of the performance analysis, a data testing comparison between the MCLP and induction decision tree approaches is demonstrated. These findings suggest that the MCLP-data mining techniques have a great potential in discovering knowledge patterns from a large-scale real-life database or data warehouse.
\end{abstract}

Keywords: Data Mining, Multi-criteria Linear Programming, Classification, Algorithm, SAS and Linux platforms

\section{Introduction}

The history of credit card can be traced back to 1951 when the Diners' Club issued the first credit card in the US to 200 customers who could use it at 27 restaurants in New York [1]. At the end of fiscal 1999, there are 1.3 billion payment cards in circulation and Americans made \$1.1 trillion credit purchases [2]. These statistics show that credit card business becomes a major power to stimulate the US economy growth in the last fifty years. However, the increasing credit card delinquencies and

\footnotetext{
" This research has been partially supported by a grant under (DUE-9796243), the National Science Foundation of USA, a National Excellent Youth Fund under (\#70028101), National Natural Science Foundation of China and a grant from the K.C. Wong Education Foundation, Chinese Academy of Sciences.
} 
personal bankruptcy rates are causing plenty of headaches for banks and other credit issuers. The increase in personal bankruptcy rates was substantial. From 1980 to 2000, the number of individual bankruptcy filings in the US increased approximately $500 \%$ [3]. How to predict bankruptcy in advance and avoid huge charge-off losses becomes a critical issue of credit card issuers. Since a credit card database can contain hundreds and thousands of credit transactions, it is impossible to discover or predict the cardholders' behaviors without using mathematical tools. In fact, the practitioners have tried a number of quantitative techniques to conduct the credit card portfolio management. Some examples of known approaches are (1) Behavior Score developed by Fair Isaac Corporation (FICO) [4]; (2) Credit Bureau Score also developed by FICO [4]; (3) First Data Resource (FDR)'s Proprietary Bankruptcy Score [5]; (4) Multiple-criteria Score [6,7] and (5) Dual-model Score [8]. A basic characteristic of these models is that they first consider the behaviors of the cardholders as two predefined classes: bankrupt accounts and non-bankrupt accounts according to their historical records. Then they use either statistical methods or neural networks to compute the Kolmogorov-Smirnov (KS) value that measures the largest separation of these two cumulative distributions of bankrupt accounts and non-bankrupt accounts in a training set [9]. The resulting KS values from the learning process are applied to the real-life credit data warehouse to predict the percentage of bankrupt accounts in the future. Thus, these methods can be generally regarded as two-group classification models in credit card portfolio management.

In order to discover more knowledge for advanced credit card portfolio management, multi-group (group number is larger than two) data mining methods are needed. Comparing with two-group classification, the multi-group classifier enlarges the difference between bankrupt accounts and non-bankrupt accounts. This enlargement increases not only the accuracy of separation, but also provides more useful information for credit card issuers or banks. From theoretical point of view, a general model of multi-group classifier is easy to construct. From practical point of view, the best control parameters (such as class boundaries) have to be identified through a learning process on a training data set. Therefore, finding the practical technology with certain size of multi-group classifier is not trivial task. Peng et al [10] and Shi et al [7] have explored a three-group classifier considering the number of months where the account has been overlimit. This model produces the prediction distribution for each behavior class and inner relationship between these classes so that credit card issuers can establish their credit limit policies for various cardholders.

The purpose of this paper is to build a set of data mining models to classify credit cardholders' behavior. Then these models are tested on the real-life data for five groups of credit cardholders who are predefined as: Bankrupt charge-off accounts, Non-bankrupt charge-off accounts, Delinquent accounts, Current accounts and Outstanding accounts. Bankrupt charge-off accounts are accounts that have been written off by credit card issuers because of cardholders' bankrupt claims. Nonbankrupt charge-off accounts are accounts that have been written off by credit card issuers due to reasons other than bankrupt claims. The charge-off policy may vary among authorized institutions. 


\section{Models of Multiple Criteria Linear Programming Classification}

A general problem of data classification by using multiple criteria linear programming can be described as [11]:

Given a set of $r$ variables or attributes in database $\boldsymbol{a}=\left(a_{1}, \ldots, a_{r}\right)$, let $\boldsymbol{A}_{i}=\left(A_{i 1}, \ldots\right.$, $\left.A_{i r}\right) \in \boldsymbol{R}^{r}$ be the sample observations of data for the variables, where $i=1, \ldots, n$ and $n$ is the sample size. If a given problem can be predefined as $s$ different classes, $\mathrm{G}_{1}, \ldots, \mathrm{G}_{\mathrm{s}}$, then the boundary between the $j t h$ and $j+1$ th classes can be $b_{j}, j=1, \ldots$, $s-1$. We want to determine the coefficients for an appropriate subset of the variables, denoted by $\boldsymbol{X}=\left(x_{1}, \ldots, x_{r}\right)^{\mathrm{T}} \in \boldsymbol{R}^{r}$ and scalars $b_{j}$ such that the separation of these classes can be described as follows:

$\boldsymbol{A}_{i} \boldsymbol{X} \leq b_{1}, \boldsymbol{A}_{i} \in \mathrm{G}_{1} ; b_{k-1} \leq \boldsymbol{A}_{i} \boldsymbol{X} \leq b_{k}, \boldsymbol{A}_{i} \in \mathrm{G}_{\mathrm{k}}, k=2, \ldots, s-1 ; \boldsymbol{A}_{i} \boldsymbol{X} \geq b_{s-1}$, $\boldsymbol{A}_{i} \in \mathrm{G}_{\mathrm{s}}$; where $\boldsymbol{A}_{i} \in \mathrm{G}_{j}, j=1, \ldots, s$, means that the data case $\boldsymbol{A}_{i}$ belongs to the class $\mathrm{G}_{j}$.

The quality of classification is measured by minimizing the total overlapping of data and maximizing the distances of every data to its class boundary simultaneously. Let $\alpha_{i}^{j}$ be the overlapping degree with respect of data case $\boldsymbol{A}_{i}$ within $\mathrm{G}_{\mathrm{j}}$ and $\mathrm{G}_{\mathrm{j}+1}$, and $\beta_{i}^{j}$ be the distance from $\boldsymbol{A}_{i}$ within $\mathrm{G}_{\mathrm{j}}$ and $\mathrm{G}_{\mathrm{j}+1}$ to its adjusted boundaries. A multiple criteria linear programming (MCLP) classification model can be defined as: (M1) Minimize $\Sigma_{i} \Sigma_{j} \alpha_{i}^{j}$ and Maximize $\Sigma_{i} \Sigma_{j} \beta_{i}^{j}$

Subject to: $\boldsymbol{A}_{i} \boldsymbol{X} \leq b_{1}+\alpha_{i}^{1}, \boldsymbol{A}_{i} \in \mathrm{G}_{1} ; b_{k-1}-\alpha_{i}^{k-1} \leq \boldsymbol{A}_{i} \boldsymbol{X} \leq b_{k}+\alpha_{i}^{k}, \boldsymbol{A}_{i} \in \mathrm{G}_{\mathrm{k}}$, $k=2, \ldots, s-1 ; \boldsymbol{A}_{i} \boldsymbol{X} \geq b_{s-1}-\alpha_{i}^{s-1}, \boldsymbol{A}_{i} \in \mathrm{G}_{\mathrm{s}} ; b_{k-1}+\alpha_{i}^{k-1} \leq b_{k}-\alpha_{i}^{k}, k=2, \ldots, s-1$, where $\boldsymbol{A}_{i}$ are given; $\boldsymbol{X}$ and $b_{j}$ are unrestricted; and $\alpha_{i}^{j}, \beta_{i}^{j} \geq 0$, for $j=1, \ldots, s-1, i=1, \ldots$, $n$.

Note that the constraints $b_{k-1}+\alpha_{i}^{k-1} \leq b_{k}-\alpha_{i}^{k}$ ensure the existence of the boundaries. If minimizing the total overlapping of data, maximizing the distances of every data to its class boundary, or a given combination of both criteria is considered separately, model (M1) is reduced to linear programming (LP) classification (known as linear discriminant analysis), which is initiated by Freed and Glover [12]. However, the single criterion LP could not determine the "best tradeoff" of two misclassification measurements. Therefore, the model (M1) is potentially better than LP classification in identifying the best tradeoff of the misclassifications for data separation. To facilitate the computation on the real-life data, a compromise solution approach [13,14,15] is employed to reform model (M1) for the "best tradeoff" between $\Sigma_{i} \Sigma_{j} \alpha_{i}^{j}$ and $\Sigma_{i} \Sigma_{j} \beta_{i}^{j}$. Let us assume the "ideal values" for $s-1$ classes overlapping $\left(-\Sigma_{i} \alpha_{i}^{1}, \ldots,-\Sigma_{i} \alpha_{i}^{s-1}\right)$ be $\left(\alpha_{*}^{1}, \ldots, \alpha_{*}^{s-1}\right)>0$, and the "ideal values" of 
$\left(\Sigma_{i} \beta_{i}^{1}, \ldots, \Sigma_{i} \beta_{i}^{s-1}\right)$ be $\left(\beta_{*}^{1}, \ldots, \beta_{*}^{s-1}\right)$. When $-\Sigma_{i} \alpha_{i}^{j}>\alpha_{*}^{j}$, we define the regret measure as $-\mathrm{d}_{\alpha j}^{+}=\alpha_{*}^{j}+\Sigma_{i} \alpha_{i}^{j}$; otherwise, it is 0 , where $j=1, \ldots, s$ - When $\Sigma_{i} \alpha_{i}^{j}<\alpha_{*}^{j}$, we define the regret measure as $\mathrm{d}_{\alpha j}^{-}=\alpha_{*}^{j}+\Sigma_{i} \alpha_{i}^{j}$; otherwise, it is 0 , where $j=1, \ldots, s-1$. Thus, we have:

Theorem 1. $\alpha_{*}^{j}+\Sigma_{i} \alpha_{i}^{j}=d_{\alpha j}^{-}-d_{o j}^{+},\left|\alpha_{*}^{j}+\Sigma_{i} \alpha_{i}^{j}\right|=d_{\alpha j}^{-}+d_{\alpha j}^{+}$, and $d_{o j}^{-}, d_{o j}^{+} \geq$ $0, j=1, \ldots, s-1$.

Similarly, we can derive:

Corollary 1. $\beta_{*}^{j}-\Sigma_{i} \beta_{i}^{j}=d_{\beta j}^{-}-d_{\beta j}^{+},\left|\beta_{*}^{j}-\Sigma_{i} \beta_{i}^{j}\right|=d_{\beta j}^{-}+d_{\beta j}^{+}$, and $d_{\beta j}^{-}, d_{\beta j}^{+} \geq 0$, $j=1, \ldots, s-1$.

Incorporating the above results into model (M1), it is reformulated as:

(M2) Minimize $\sum_{j=1}^{s-1}\left(d_{\alpha j}^{-}+d_{\alpha j}^{+}+d_{\beta j}^{-}+d_{\beta j}^{+}\right)$

Subject to: $\alpha_{*}^{j}+\Sigma_{i} \alpha_{i}^{j}=d_{\alpha j}^{-}-d_{\alpha j}^{+}, j=1, \ldots, s-1 ; \beta_{*}^{j}-\Sigma_{i} \beta_{i}^{j}=d_{\beta j}^{-}-d_{\beta j}^{+}, j=1, \ldots$, $s-1$;

$\boldsymbol{A}_{i} \boldsymbol{X} \leq b_{1}+\alpha_{i}^{1}, \boldsymbol{A}_{i} \in \mathrm{G}_{1} ; b_{k-1}-\alpha_{i}^{k-1} \leq \boldsymbol{A}_{i} \boldsymbol{X} \leq b_{k}+\alpha_{i}^{k}, \boldsymbol{A}_{i} \in \mathrm{G}_{\mathrm{k}}, k=2, \ldots, s-1 ;$

$\boldsymbol{A}_{i} \boldsymbol{X} \geq b_{s-1}-\alpha_{i}^{s-1}, \boldsymbol{A}_{i} \in \mathrm{G}_{\mathrm{s}} ; b_{k-1}+\alpha_{i}^{k-1} \leq b_{k}-\alpha_{i}^{k}, k=2, \ldots, s-1$,

where $\boldsymbol{A}_{i}, \alpha_{*}^{j}$, and $\beta_{*}^{j} \quad$ are given; $\boldsymbol{X}$ and $b_{j}$ are unrestricted; and $\alpha_{i}^{j}, \beta_{i}^{j}, d_{\alpha j}^{-}, d_{\alpha j}^{+}$, $d_{\beta j}^{-}, d_{\beta j}^{+} \geq 0$, for $j=1, \ldots, s-1, i=1, \ldots, n$.

We can call model (M1) or (M2) is a "weak separation formula" since it considers as many overlapping data as possible. We can build a "medium separation formula" on the absolute class boundaries in (M3) and a "strong separation formula" which contains as few overlapping data as possible in (M4).

(M3) Minimize $\sum_{j=1}^{s-1}\left(d_{\alpha j}^{-}+d_{\alpha j}^{+}+d_{\beta j}^{-}+d_{\beta j}^{+}\right)$

Subject to: $\alpha_{*}^{j}+\Sigma_{i} \alpha_{i}^{j}=d_{\alpha j}^{-}-d_{\alpha j}^{+}, j=1, \ldots, s-1 ; \beta_{*}^{j}-\Sigma_{i} \beta_{i}^{j}=d_{\beta j}^{-}-d_{\beta j}^{+}, j=1, \ldots$, $s-1$;

$\boldsymbol{A}_{i} \boldsymbol{X} \leq b_{1}, \boldsymbol{A}_{i} \in \mathrm{G}_{\mathrm{l}} ; b_{k-1} \leq \boldsymbol{A}_{i} \boldsymbol{X} \leq b_{k}, \boldsymbol{A}_{i} \in \mathrm{G}_{\mathrm{k}}, k=2, \ldots, s-1 ;$

$\boldsymbol{A}_{i} \boldsymbol{X} \geq b_{s-1}, \boldsymbol{A}_{i} \in \mathrm{G}_{\mathrm{s}} ; b_{k-1}+\varepsilon \leq b_{k}-\alpha_{i}^{k}, k=2, \ldots, s-1$, 
where $\boldsymbol{A}_{i}, \varepsilon, \alpha_{*}^{j}$, and $\beta_{*}^{j} \quad$ are given; $\boldsymbol{X}$ and $b_{j}$ are unrestricted; and $\alpha_{i}^{j}, \beta_{i}^{j}, d_{\alpha j}^{-}, d_{\alpha j}^{+}$, $d_{\beta j}^{-}, d_{\beta j}^{+} \geq 0$, for $j=1, \ldots, s-1, i=1, \ldots, n$.

(M4) Minimize $\sum_{j=1}^{s-1}\left(d_{\alpha j}^{-}+d_{\alpha j}^{+}+d_{\beta j}^{-}+d_{\beta j}^{+}\right)$

Subject to: $\alpha_{*}^{j}+\Sigma_{i} \alpha_{i}^{j}=d_{\alpha j}^{-}-d_{\alpha j}^{+}, j=1, \ldots, s-1 ; \beta_{*}^{j}-\Sigma_{i} \beta_{i}^{j}=d_{\beta j}^{-}-d_{\beta j}^{+}, j=1, \ldots$, $s-1$

$\boldsymbol{A}_{i} \boldsymbol{X} \leq b_{1}-\alpha_{i}^{1}, \boldsymbol{A}_{i} \in \mathrm{G}_{1} ; b_{k-1}+\alpha_{i}^{k-1} \leq \boldsymbol{A}_{i} \boldsymbol{X} \leq b_{k}-\alpha_{i}^{k}, \boldsymbol{A}_{i} \in \mathrm{G}_{\mathrm{k}}, k=2, \ldots, s-1$;

$\boldsymbol{A}_{i} \boldsymbol{X} \geq b_{s-1}+\alpha_{i}^{s-1}, \boldsymbol{A}_{i} \in \mathrm{G}_{s} ; b_{k-1}+\alpha_{i}^{k-1} \leq b_{k}-\alpha_{i}^{k}, k=2, \ldots, s-1$,

where $\boldsymbol{A}_{i}, \alpha_{*}^{j}$, and $\beta_{*}^{j} \quad$ are given; $\boldsymbol{X}$ and $b_{j}$ are unrestricted; and $\alpha_{i}^{j}, \beta_{i}^{j}, d_{\alpha j}^{-}, d_{\alpha j}^{+}$, $d_{\beta j}^{-}, d_{\beta j}^{+} \geq 0$, for $j=1, \ldots, s-1, i=1, \ldots, n$.

A loosing relationship of models (M2), (M3), and (M4) is given as:

Theorem 2. If a data case $\boldsymbol{A}_{i}$ is classified in a given class $\mathrm{G}_{\mathrm{j}}$ by model (M4), then it may be in $\mathrm{G}_{\mathrm{j}}$ by using models (M3) and (M2). If a data case $\boldsymbol{A}_{i}$ is classified in a given class $\mathrm{G}_{\mathrm{j}}$ by model (M3), then it may be in $\mathrm{G}_{\mathrm{j}}$ by using models (M2).

Example 1. As an illustration, we use a small training data set adapted from [16,17] in Table 1 (Column 1-6) to show how the two-class model works.

Suppose whether or not a customer buys computer relates to the attribute set Age, Income, Student and Credit_rating\}. We first define the variables Age, Income, Student and Credit_rating by numeric numbers as follows:

For Age: " $\leq 30$ " assigned to be " 3 "; " $31 \ldots 40$ " to be " 2 "; and " $>40$ " to be " 1 ".

For Income: "high" assigned to be " 3 "; "medium" to be "2"; and "low" to be "1".

For Student: "yes" assigned to be " 2 " and "no" to be " 1 ".

For Credit_rating: "excellent" assigned to be " 2 " and "fair" to be " 1 ".

$\mathrm{G} 1=\{$ yes to buys_computer $\}$ and G2 $=\{$ no to buys_computer $\}$

Then, let $j=1,2$ and $i=1, \ldots, 14$, model (M2) for this problem to classify the customer's status for \{buys_computer $\}$ is formulated by

Minimize $d_{\alpha}{ }^{-}+d_{\alpha}{ }^{+}+d_{\beta}{ }^{-}+d_{\beta}^{+}$

Subject to:

$\alpha^{*}+\Sigma_{i} \alpha_{i}=d_{\alpha}{ }^{-}-d_{\alpha}{ }^{+}, \beta^{*}-\Sigma_{i} \beta_{i}=d_{\beta}{ }^{-}-d_{\beta}{ }^{+}$,

$2 x_{1}+3 x_{2}+x_{3}+x_{4}=b+\alpha_{1}-\beta_{1}, x_{1}+2 x_{2}+x_{3}+x_{4}=b+\alpha_{2}-\beta_{2}$

$x_{1}+x_{2}+2 x_{3}+x_{4}=b+\alpha_{3}-\beta_{3}, 2 x_{1}+x_{2}+2 x_{3}+2 x_{4}=b+\alpha_{4}-\beta_{4}$

$3 x_{1}+x_{2}+2 x_{3}+x_{4}=b+\alpha_{5}-\beta_{5}, x_{1}+2 x_{2}+2 x_{3}+x_{4}=b+\alpha_{6}-\beta_{6}$

$3 x_{1}+2 x_{2}+2 x_{3}+2 x_{4}=b+\alpha_{7}-\beta_{7}, 2 x_{1}+2 x_{2}+x_{3}+2 x_{4}=b+\alpha_{8}-\beta_{8}$

$2 x_{1}+3 x_{2}+2 x_{3}+x_{4}=b+\alpha_{9}-\beta_{9}, 3 x_{1}+3 x_{2}+x_{3}+x_{4}=b+\alpha_{10}-\beta_{10}$ 
$3 x_{1}+3 x_{2}+x_{3}+2 x_{4}=b+\alpha_{11}-\beta_{11}, x_{1}+x_{2}+2 x_{3}+2 x_{4}=b+\alpha_{12}-\beta_{12}$

$3 x_{1}+2 x_{2}+x_{3}+x_{4}=b+\alpha_{13}-\beta_{13}, x_{1}+2 x_{2}+x_{3}+2 x_{4}=b+\alpha_{14}-\beta_{14}$

where $\alpha^{*}$, and $\beta^{*}$ are given, $x_{1}, x_{2}, x_{3}, x_{4}$ and $b$ are unrestricted, and $\alpha_{i}, \beta_{i} d_{\alpha}$, $d_{\alpha}{ }^{+}, d_{\beta}{ }^{-}, d_{\beta}{ }^{+} \geq 0, i=1, \ldots, 14$.

Before solving the above problem for data classification, we have to choose the values for the control parameters $\alpha^{*}, \beta^{*}$ and $b$. Suppose we use $\alpha^{*}=0.1, \beta^{*}=$ 30000 and $b=1$. Then, the optimal solution of this linear program for the classifier is obtained as Column 7 of Table 1, where only cases $\boldsymbol{A}_{8}$ and $\boldsymbol{A}_{12}$ are misclassified. In other words, cases $\left\{\boldsymbol{A}_{1}, \boldsymbol{A}_{2} \boldsymbol{A}_{3}, \boldsymbol{A}_{4}, \boldsymbol{A}_{5}, \boldsymbol{A}_{6}, \boldsymbol{A}_{7}, \boldsymbol{A}_{9}\right\}$ are correctly classified in G1, while cases $\left\{\boldsymbol{A}_{10}, \boldsymbol{A}_{11}, \boldsymbol{A}_{13}, \boldsymbol{A}_{14}\right\}$ are found in G2. Similarly, when we apply models (M3) and (M4) with $\varepsilon=0$, one of learning processes provides the same results where cases $\left\{\boldsymbol{A}_{1}, \boldsymbol{A}_{2}, \boldsymbol{A}_{3}, \boldsymbol{A}_{5}, \boldsymbol{A}_{8}\right\}$ are correctly classified in G1, while cases $\left\{\boldsymbol{A}_{10}, \boldsymbol{A}_{11}, \boldsymbol{A}_{12}, \boldsymbol{A}_{14}\right\}$ are correctly found in G2. Then, we see that cases $\left\{\boldsymbol{A}_{1}, \boldsymbol{A}_{2}, \boldsymbol{A}_{3}, \boldsymbol{A}_{5}\right\}$ classified in $\mathrm{G} 1$ by model (M4) are also in G1 by models (M3) and (M2), and cases $\left\{\boldsymbol{A}_{10}, \boldsymbol{A}_{11}, \boldsymbol{A}_{14}\right\}$ classified in G2 by model (M4) are in G2 by model (M3) and (M2). This is consistent to Theorem 2 .

Table 1. A two-class data set of customer status

\begin{tabular}{|c|c|c|c|c|c|c|}
\hline Cases & Age & Income & Student & $\begin{array}{l}\text { Credit } \\
\text { Rating }\end{array}$ & $\begin{array}{l}\text { Class: } \\
\text { buys_- } \\
\text { computer }\end{array}$ & $\begin{array}{l}\text { Training } \\
\text { results }\end{array}$ \\
\hline$A_{1}$ & $31 \ldots 40$ & high & no & fair & yes & success \\
\hline$A_{2}$ & $>40$ & medium & no & fair & yes & success \\
\hline$A_{3}$ & $>40$ & low & yes & fair & yes & success \\
\hline$A_{4}$ & $31 \ldots 40$ & low & yes & excellent & yes & success \\
\hline$A_{5}$ & $\leq 30$ & low & yes & fair & yes & success \\
\hline$A_{6}$ & $>40$ & medium & yes & fair & yes & success \\
\hline $\boldsymbol{A}_{7}$ & $\leq 30$ & medium & yes & excellent & yes & success \\
\hline$A_{8}$ & $31 \ldots 40$ & medium & no & excellent & yes & failure \\
\hline$A_{9}$ & $31 \ldots 40$ & high & yes & fair & yes & success \\
\hline$A_{10}$ & $\leq 30$ & high & no & fair & no & success \\
\hline$A_{11}$ & $\leq 30$ & high & no & excellent & no & success \\
\hline$A_{12}$ & $>40$ & low & yes & excellent & no & failure \\
\hline$A_{13}$ & $\leq 30$ & medium & no & fair & no & success \\
\hline$A_{14}$ & $>40$ & medium & no & excellent & no & success \\
\hline
\end{tabular}

\section{Algorithm Implementation and Software Development}

A general algorithm to execute the MCLP classification method can be outlined as:

\section{Algorithm 1.}

Step 1 Build a data mart for task data mining project.

Step 2 Generate a set of relevant attributes or dimensions from a data mart, transform the scales of the data mart into the same numerical measurement and determine predefined classes, classification threshold, training set and verifying set. Step 3 Use the MCLP model to learn and compute the best overall score $\left(\boldsymbol{X}^{*}\right)$ of the relevant attributes or dimensions over all observations.

Step 4 Discover the interested patterns that can best match the original classes under the threshold by choosing the proper control parameters $\left(\alpha^{*}, \beta^{*}\right.$ and $\left.b\right)$. If the patterns are found, go to Step 5. Otherwise, go back to Step 3.

Step 5 Apply the final learned score $\left(\boldsymbol{X}^{* *}\right)$ to predict the unknown data cases. 
Two versions of actual software have been developed for the MCLP classification method. The first version is based on the well-known commercial SAS platform. In this software, we have applied SAS codes to execute Algorithm 1 in which the MCLP models (M2)-(M4) utilize SAS linear programming procedure. The second version of the software is written by $\mathrm{C}++$ language running on Linux platform. The reason for developing Linux version of the MCLP classification software is that the majority of database vendors, such as IBM are aggressively moving to Linux-based system development. Our Linux version goes along with the trend of information technology. Because many large companies currently use SAS system for data analysis, our SAS version is also useful to conduct data mining analysis under SAS environment.

\section{Experimental Results from a Real-Life Database}

Given a set of attributes, such as monthly payment, balance, purchase, and cash advance and the criteria about "bankruptcy", the purpose of data mining in credit card portfolio management is to find the better classifier through a training set and use the classifier to predict all other customer's spending behaviors [18]. The frequently used data-mining model in the business is still two-class separation technique. The key of two-class separation is to separate the "bankruptcy" accounts from the "current" accounts and identify as many bankruptcy accounts as possible. This is also known as the method of "making black list." The examples of popular methods are Behavior Score, Credit Bureau Score, FDC Bankruptcy Score, and Set Enumeration Decision Tree Score [6]. These methods were developed by either statistics or decision tree. Using a terabyte real credit database of the major US Bank, the SAS version of two-class MCLP model (as in Example) has demonstrated a better predication power (e.g., higher KS values) than these popular business methods [6].

\subsection{Testing on Five-Class MCLP Models}

We demonstrate the experimental results of 5-class MCLP model in Linux version on a real-life credit data warehouse from a major US bank. Since this database contains 64 attributes with a lot of overlapping, we employed the weak separation model (M2).

In the five-class MCLP model, we defined five classes as Bankrupt charge-off accounts (the number of over-limits $\geq 13$ ), Non-bankrupt charge-off accounts $(7 \leq$ the number of over-limits $\leq 12)$, Delinquent accounts $(3 \leq$ the number of over-limits $\leq 6)$, Current accounts ( $1 \leq$ the number of over-limits $\leq 2)$, and Outstanding accounts (no over limit). We selected 200 samples with 40 accounts in each class as the training set. The same 5,000 samples with 53 in G1; 165 in G2; 379 in G3, 482 in G4 and 3921 in G5 were used as the verifying set. We found, in the training process, that G1 has been correctly identified $47.5 \%$ (19/40), G2 55\% (22/40), G3 47.5\% (19/40), G4 42.5\% (17/40), and KS values 42.5 for G1 vs. G2, G2 vs. G3 and G3 vs. G4, but 67.5 for G4 vs. G5 in Figure 1. When we used this as the better classifier, we predicted the verifying set as G1 for $50.9 \%$ (27/53), G2 for $49.7 \%(82 / 165)$, G3 for $40 \%(150 / 379), \mathrm{G} 4$ for $31.1 \%(150 / 482)$ and G5 for $54.6 \%(2139 / 3921)$. The predicted KS values are 36.08 for G1 vs. G2, 23.3 for G2 vs. G3, 27.82 for G3 vs. 
G4, and 42.17 for G4 vs. G5 in Figure 2. This indicates that the separation between G4 and G5 is better than other situations. In other words, the classifier is favorable to G4 vs. G5. We note that many real-life applications do not require more than five classes separations. This claim is partially supported by psychological studies. According to [19], Human attention span is "seven plus or minus two". Therefore, for the practical purpose of classification in data mining, classifying five interesting classes in a terabyte database can be very meaningful.

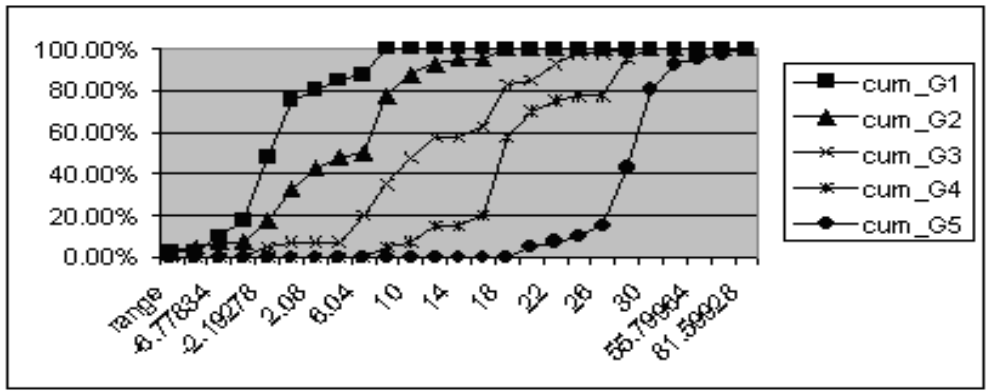

Fig. 1. Five-Class Training Data Set

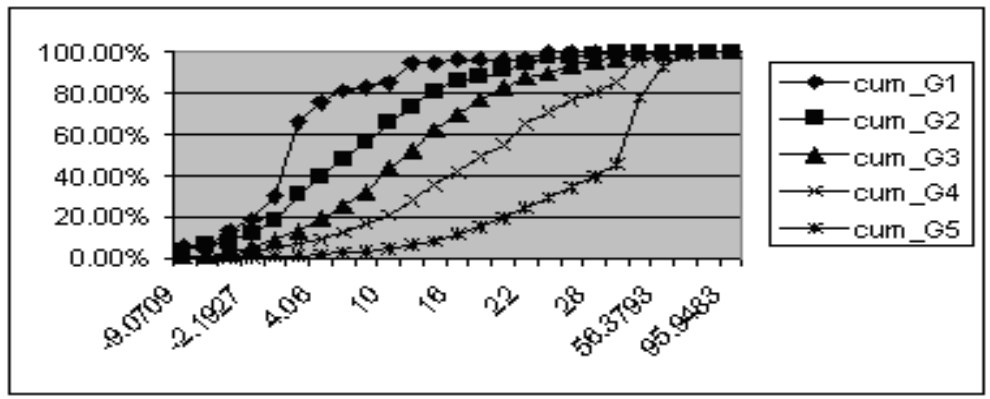

Fig. 2. Five-Class Verifying Data Set

\subsection{Comparison of MCLP Method and Decision Tree Method}

A commercial Decision Tree software C5.0 (the newly updated version of C4.5) was used to test the classification accuracy of the multiple classes (from two to five) against MCLP methods [17, 20]. Given a terabyte credit card database of the major US Bank, the number samples for the training sets were 600 for two-class problem, 300 for three-class problem, 160 for four-class problem, and 200 for five-class problem. The verifying sets were used the same 5,000 credit card records as in Section 4.1. Table 2 summarizes these comparisons of two methods. As we see, generally the decision tree method does a better job than the MCLP method on training sets when the sample size is small. When applying the classifier from the 
training process on the larger verifying sets, the MCLP method outperforms the decision tree method. Two issues may cause this evidence. One is that the MCLP method as a linear model may miss some nonlinear nature of data, while the decision tree is nonlinear model. This could be the reason why the latter is better than the former in the training process. However, the robustness and stability of the MCLP is better than the decision tree when the classifier is applied to predict the classification of the verifying sets. This may be due to the fact that the MCLP method employs optimization to find the optimal factors from all feasible factors for the scores while the decision tree method just selects the better tree from a limited built trees, which is not a best tree. In addition, when the decision tree gets big (i.e., the size of the verifying sets increase), the pruning procedure may further eliminate some better branches.

Table 2. Five-class Comparison of MCLP and Decision Tree

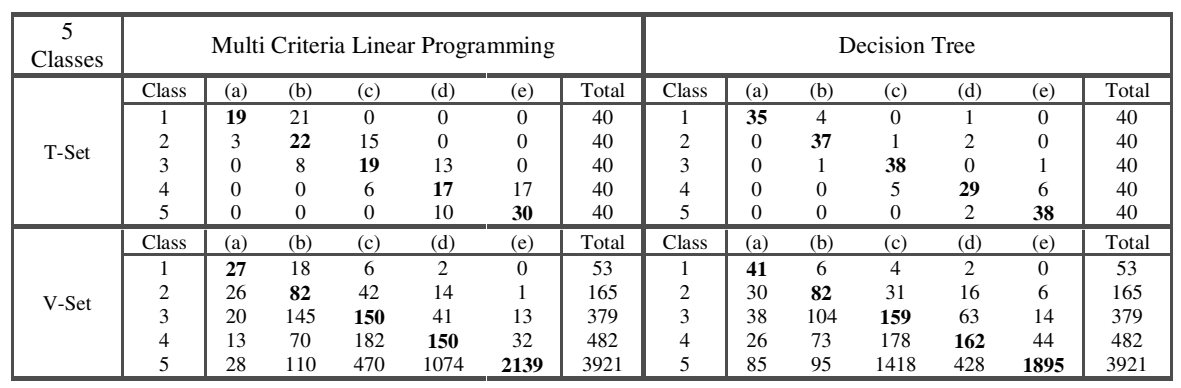

Furthermore, a parallel experimental study on the MCLP classifications through the developed SAS version can be referred to [18]. For the sake of space, we will not elaborate on the results here.

\section{Concluding Remarks}

There are some research and experimental problems remaining to be explored. From the structure of MCLP formulation, the detailed theoretical relationship of models (M2), (M3), and (M4) need to be further investigated in terms of classification separation accuracy and predictive power. In addition, in the proposed MCLP models, the penalties to measure the "cost" of misclassifications (or coefficients of $\Sigma_{i} \Sigma_{j} \alpha_{i}^{j}$ and $\Sigma_{i} \Sigma_{j} \beta_{i}^{j}$ ) were fixed as 1 . If they are allowed to change, their influence on the classification results can be studied, and a theoretical sensitivity analysis of the misclassification in the MCLP models will be also conducted. From mathematical structure point of view, a multiple criteria non-linear classification model may be generalized if the hyper-plane $\boldsymbol{X}$ becomes non-linear cases, say $\boldsymbol{X}^{p}, p$ $>1$. The possible connection of the MCLP classification with the known Support Vector Machine (SVM) method in pattern recognition can be researched. In the empirical tests, we have noticed that identifying the optimal solution for model (M2), (M3), or (M3) in the training process may be time-consuming. Instead, we can apply the concept of fuzzy multiple criteria linear programming to seek a satisfying 
solution that may lead to a better data separation. Other well-known methods, such as neural networks, rough set, and fuzzy set should be considered into part of the extensive comparison study against the MCLP method so that the MCLP method can be known in the data mining community for both researchers and practitioners. We will report any significant results from these ongoing projects in the near future.

\section{References}

1 The First Credit Card Was Issued in 1951. http://www.didyouknow.cd/creditcards.htm.

2 Debt statistics. http://www.nodebt.org/debt.ntm.

3 Stavins, J.: Cređit Card Borrowing, Delinquency, And Personal Bankruptcy. New England Economic Review. July/August 2000, http://www.bos.frb.org/economic/neer/neer2000/neer400b.pdf.

4 Www.fairisaac.com

5 http://www.tirstdatacorp.com

6 Shi, Y., Wise, M., Luo, M., Lin, Y.: Data Mining in Credit Card Portfolio Management: A Multiple Criteria Decision Making Approach. In: Koksalan, M., Zionts, S. (eds.): Multiple Criteria Decision Making in the New Millennium. Springer, Berlin (2001) 427436.

7 Shi, Y., Peng, Y., Xu, W., Tang, X.: Data Mining Via Multiple Criteria Linear Programming: Applications in Credit Card Portfolio Management. International Journal of Information Technology and Decision Making. 1 (2002) 131-151.

8 Lin, Y.: Improvement On Behavior Scores by Dual-Model Scoring System. International

9 Journal of Information Technology and Decision Making. 1 (2002) 153-164.

10 Conover, W.J.: Practical Nonparametric Statistics. Wiley (1999).

11 Peng, Y., Shi, Y., Xu, W: Classification for Three-Group Of Credit Cardholders' Behavior Via A Multiple Criteria Approach. Advanced Modeling and Optimization. 4 (2002) 39-56.

12 Kou, G., Peng, Y., Shi, Y., Wise M., Xu, W.: Discovering Credit Cardholders' Behavior by Multiple Criteria Linear Programming. Working Paper, College of Information Science and Technology, University of Nebraska at Omaha (2002).

13 Freed, N., Glover, F.: Simple but Powerful Goal Programming Models for Discriminant Problems. European Journal of Operational Research. 7 (1981) 44-60.

14 Shi, Y., Yu, P.L.: Goal Setting and Compromise Solutions. In Karpak, B., Zionts, S. (eds.): Multiple Criteria Decision Making and Risk Analysis Using Microcomputers. Springer-Verlag, Berlin (1989) 165-204.

15 Shi, Y.: Multiple Criteria Multiple Constraint-levels Linear Programming: Concepts, Techniques and Applications. World Scientific Publishing, River Edge, New Jersey (2001).

16 Yu, P.L.: Multiple Criteria Decision Making: Concepts, Techniques and Extensions. Plenum, New York (1985).

17 Han, J., Kamber, M.: Data Mining: Concepts and Techniques. Morgan Kaufmann Publishers, San Francisco, California (2001).

18 Quinlan, J.: Induction of Decision Trees. Machine Learning. 1 (1986) 81-106.

19 Peng, Y.: Data Mining in Credit Card Portfolio Management: Classifications for Card Holder Behavior. Master Thesis, College of Information Science and Technology, University of Nebraska at Omaha (2002).

20 Miller, G.A.: The Magical Number Seven, Plus or Minus Two: Some Limits on Our Capacity for Processing Information. The Psychological Review. 63 (1956) 81-97.

21 nttp://www.rulequest.com/sees-info.htm! 